\title{
Infección por el virus de la hepatitis C en individuos transfundidos antes de 1994 en Antioquia, Colombia
}

\section{Hepatitis C Infections among Individuals who Received Transfusions prior to 1994 in Antioquia, Colombia}

Johanna C. Arroyave 0, ${ }^{1}$ Claudia Álvarez F, ${ }^{2}$ Gonzalo Correa A, ${ }^{2}$ Norman Balcázar M, ${ }^{3}$ María Patricia Arbeláez M, ${ }^{4}$ María Cristina Navas N. ${ }^{2}$

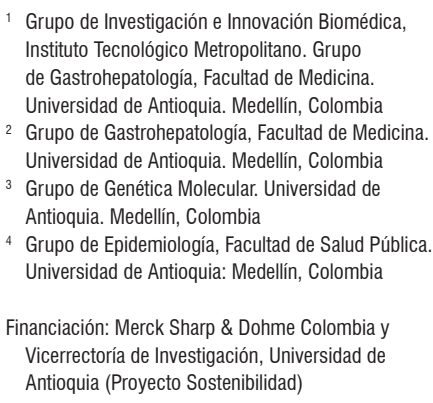

Grupo de Investigación e Innovación Biomédica, Instituto Tecnológico Metropolitano. Grupo de Gastrohepatología, Facultad de Medicina. Universidad de Antioquia. Medellín, Colombia 2 Grupo de Gastrohepatología, Facultad de Medicina. Universidad de Antioquia. Medellín, Colombia

3 Grupo de Genética Molecular. Universidad de Antioquia. Medellín, Colombia

${ }^{4}$ Grupo de Epidemiología, Facultad de Salud Pública. Universidad de Antioquia: Medellín, Colombia

Financiación: Merck Sharp \& Dohme Colombia y Vicerrectoría de Investigación, Universidad de Antioquia (Proyecto Sostenibilidad)

Presentación en eventos: 10th International Meeting on Hepatitis C Virus and Related Viruses. Kyoto, Japan Dec 2-6, 2003.

Fecha recibido: $\quad 08-05-14$ Fecha aceptado: 05-11-14

\begin{abstract}
Resumen
Introducción: la infección por el virus de la hepatitis $\mathrm{C}$ es un problema de salud pública. Según datos de la Organización Mundial de la Salud, se estiman 184 millones de casos de infección por VHC en el mundo. El principal factor de riesgo en países en desarrollo corresponde a la transfusión de componentes sanguíneos. En Colombia, en 1993, se reglamentó el tamizaje serológico en los bancos de sangre para diferentes agentes infecciosos, incluido el VHC; sin embargo, los datos de infección por VHC en la población transfundida antes de esta fecha es limitada.

Objetivo: describir la frecuencia de infección por el VHC en una población de individuos transfundidos antes de 1994 en Antioquia.

Materiales y Métodos: un total de 166 individuos transfundidos antes de 1994 aceptaron participar en el estudio. A partir de las muestras de suero se realizó la detección de anticuerpos totales contra el VHC (antiVHC) mediante prueba de ELISA y en las muestras positivas se determinó la presencia del genoma viral por RT-PCR de la región no codificante 5'.

Resultados y conclusiones: en el población de estudio se encontró una frecuencia de anticuerpos antiVHC de 6,6\% (11/166) y presencia del genoma del VHC en 7/11 de las muestras; el genotipo 1 se identificó en 4 de las muestras. No se encontró asociación de otros factores de riesgo diferentes a transfusión en los individuos con marcadores de infección por el VHC. Este estudio aporta datos a la epidemiología de la infección por el VHC en Colombia.
\end{abstract}

\section{Palabras clave}

Virus de la hepatitis C, ELISA, genotipo, transfusión de componentes sanguíneos.

\section{Abstract}

Introduction: Infection with the hepatitis $C$ virus is a public health problem. According to the World Health Organization there are an estimated 184 million cases of HCV infection around the world. The main risk factor in developing countries is transfusion of blood components. In 1993, Colombian regulations began requiring serological screening by blood banks for infectious agents including HCV. Nevertheless, data about HCV infections in the population transfused before this date is limited.

Objective: The objective of this study is to describe the frequency of HCV infection in the population of individuals transfused before 1994 in Antioquia.

Materials and Methods: A total of 166 individuals transfused before 1994 agreed to participate in the study. ELISA tests for antibodies to HCV were performed on these patients' serum samples. Samples that were positive were tested for the presence of the viral genome by RT-PCR of non-coding region 5 .

Results and Conclusions: The frequency of anti-HCV antibodies in study population was $6.6 \%(11 / 166)$ while the HCV genome was present in seven of these eleven individuals. Genotype 1 was identified in four of the samples. No associations of different risk factors for transfusion in individuals with markers of HCV infection were found. This study provides data on the epidemiology of HCV infection in Colombia.

\section{Keywords}

Hepatitis $C$ virus, ELISA, genotype, transfusion of blood components. 


\section{INTRODUCCIÓN}

La infección por el VHC constituye un problema de salud pública, con más de 184 millones de individuos infectados, lo que corresponde a cerca de $3 \%$ de la población mundial (1). La infección crónica se presenta entre $60-80 \%$ de los pacientes, quienes pueden estar en riesgo de desarrollar hepatopatías terminales como cirrosis hepática y/o carcinoma hepatocelular ( $\mathrm{CHC})(2)$.

A nivel global, entre $25-30 \%$ de los casos de CHC están asociados a la infección por VHC. Sin embargo, esta frecuencia varía dependiendo de la región geográfica; es el caso de Europa, Estados Unidos y Japón donde la infección por VHC es el factor de riesgo de 50 a $70 \%$ de los casos de CHC (3-6). En Argentina y Brasil, la infección por VHC se asocia con $30 \%$ de los casos de CHC, aproximadamente $(7,8)$.

Se estima que alrededor de 6,8 a 8,9 millones de adultos en países de Latinoamérica presentan positividad para anticuerpos contra el VHC (anti-VHC). En la mayoría de los estudios en poblaciones de Centro y Suramérica se ha demostrado que el principal factor de riesgo corresponde a transfusión de componentes sanguíneos, con excepción de los países del cono sur como Argentina y Brasil, donde se ha observado recientemente aumento del número de casos asociado al uso de drogas psicoactivas por vía endovenosa, similar a lo reportado en Europa y Estados Unidos (9).

En Colombia, en 1993, se reglamentó el tamizaje de los componentes sanguíneos para diferentes marcadores de infección (decreto 1571, agosto 1993, Ministerio de Salud), incluyendo el marcador de infección por VHC (anti-VHC). En un estudio realizado con la información suministrada por los 172 bancos de sangre del país se estableció que en el año 1995, de las 367.891 unidades analizadas, $1 \%$ fueron reactivas para anti-VHC (10). En 1997, el análisis en los bancos de sangre del departamento del Valle del Cauca demostró que 0,98\% de los donantes de sangre de la ciudad de Cali presentaron seropositividad para antiVHC, comparado con $0,47 \%$ de los donantes del resto del departamento (11). En el más reciente Informe Nacional de Indicadores, la seropositividad para VHC fue de 0,49\% en 746.059 unidades de sangre analizadas en los bancos de sangre del territorio nacional (12).

Debido al limitado número de estudios del estado de la infección por el VHC en población transfundida antes de la reglamentación del tamizaje de unidades de sangre, el presente trabajo describe retrospectivamente las principales características clínicas y demográficas en individuos transfundidos en Antioquia antes de 1994, y la frecuencia de infección por VHC utilizando marcadores serológicos y moleculares.

\section{MATERIALES Y MÉTODOS}

\section{Población y muestras}

Se realizó un estudio retrospectivo en individuos en Antioquia que declararon como antecedente al menos un episodio de transfusión de componentes sanguíneos antes de 1994. La búsqueda se realizó de manera activa en bases de datos de instituciones hospitalarias, mediante campañas radiales y visitas programadas a empresas, durante el periodo de febrero-septiembre de 2003. Una vez obtenida la firma voluntaria del consentimiento informado de 166 participantes, se diligenció un cuestionario de variables demográficas, motivo de transfusión y factores de riesgo. Una muestra de sangre total se obtuvo de cada participante por venopunción del antebrazo en condiciones asépticas; las muestras de suero fueron almacenadas a $-70^{\circ} \mathrm{C}$ hasta su procesamiento.

\section{Caracterización de la infección por el VHC}

\section{Pruebas serológicas}

Para la detección de anticuerpos totales anti-VHC, las muestras de suero fueron analizadas por duplicado mediante prueba de ELISA de tercera generación (BIOMERIEUX UBI HCV EIA), con una sensibilidad de $98,6 \%$ y una especificidad de $99,8 \%$ siguiendo las recomendaciones del estuche. Se consideró positiva una muestra cuando en las dos alícuotas analizadas fue reactiva según la razón entre la densidad óptica y el valor de corte.

\section{Detección molecular del VHC}

La detección del genoma del VHC se realizó previa extracción de ARN mediante el método de fenol-cloroformoisotiocianato de guanidina a partir de las muestras de suero que resultaron reactivas para anticuerpos totales anti-VHC (13). Posteriormente, se realizó amplificación por RT-PCR anidada, según el protocolo reportado por Chan en 1992, el cual amplifica la región no codificante 5'UTR utilizando la combinación de primers HCV940, HCV211, HCV939 y HCV209 (14). Los productos amplificados fueron visualizados mediante electroforesis en gel de agarosa al $2 \%$, teñido con bromuro de etidio para confirmar la presencia del fragmento esperado de $251 \mathrm{pb}$. Como control positivo de los ensayos se realizó la amplificación a partir del clon infeccioso HCV-H77 genotipo 1a (donado gentilmente por el doctor Charles Rice, The Rockefeller University, New York).

\section{Análisis de genotipificación mediante RFLP}

Con el fin de describir el genotipo viral en las muestras analizadas, se realizó el análisis de polimorfismos de los 
productos de amplificación y restricción con las enzimas HaeIII, RsaI, MvaI y HinfI durante 2 horas a $37^{\circ} \mathrm{C}$ con las enzimas. El patrón de restricción RFLP (siglas en inglés RFLP, Restriction Fragment Length Polymorphism) fue identificado mediante electroforesis en gel de agarosa al $4 \%$ según lo publicado previamente (15).

\section{ANÁLISIS ESTADÍSTICO}

Las variables cuantitativas se describieron como mediana y rango intercuartílico (RIQ), las variables cualitativas como números absolutos y porcentajes. Para comparar variables cualitativas se empleó la prueba de Chi cuadrado. Para comparar variables cuantitativas se utilizó la prueba $U$ de Mann-Whitney. Se asumió un valor de $\mathrm{p}<0,05$ como significativamente estadístico. Para estos análisis se utilizó el paquete estadístico SPSS 10.0.

\section{RESULTADOS}

Un total de 166 individuos transfundidos antes de 1994 participaron voluntariamente en el estudio. El 69,9\% (116/166) de la población de estudio correspondió a mujeres y $30,1 \%$ (50/166) a hombres; el promedio de edad de la población de estudio fue de 42 años (RIQ 36-49), con un rango de 10-75 años. Las características demográficas de los individuos incluidos en el estudio se resumen en la tabla 1.

Tabla 1. Características demográficas y factores de riesgo de individuos transfundidos antes de 1994 en Antioquia.

\begin{tabular}{lcccccc}
\hline & \multicolumn{2}{c}{ Mujeres } & \multicolumn{2}{c}{ Hombres } & \multicolumn{2}{c}{ Total } \\
\cline { 2 - 7 } & $\mathbf{n}$ & $\%$ & $\mathbf{n}$ & $\%$ & $\mathbf{n}$ & $\%$ \\
\hline Características & 116 & $69,9 \%$ & 50 & $30,1 \%$ & 166 & $100 \%$ \\
Edad promedio & \multicolumn{2}{c}{41,84} & \multicolumn{2}{c}{41,66} & \multicolumn{2}{c}{41,78} \\
Factores de riesgo* & & & & & & \\
Tatuajes & 12 & $10,3 \%$ & 2 & $4 \%$ & 14 & $8,4 \%$ \\
Piercing & 2 & $1,7 \%$ & 3 & $6 \%$ & 5 & $3 \%$ \\
Hemodiálisis & 2 & $1,7 \%$ & 1 & $2 \%$ & 3 & $1,8 \%$ \\
\hline
\end{tabular}

* Factores de riesgo adicionales a la transfusión.

Se determinó también la presencia otros factores de riesgo incluyendo hemodiálisis $(1,8 \%)$, tatuajes $(8,4 \%)$ y piercings (3\%), los cuales se encontraron presentes en bajo porcentaje de la población estudiada. Ninguno de los individuos del estudio declaró el uso de drogas psicoactivas por vía intravenosa.

Cirugía, accidentes de tránsito, heridas con arma de fuego y exanguinotransfusión corresponden a las 4 primeras indicaciones de transfusión en la población masculina. En la población femenina del estudio, cirugía (incluyendo cesárea), hemorragia posparto, aborto y exanguinotransfusión corresponden a los más frecuentes motivos de transfusión (tabla 2). La mayoría de los pacientes $(83,1 \%)$ fue transfundido en centros hospitalarios de Medellín. El resto, en hospitales del área metropolitana y de municipios del departamento de Antioquia; con excepción de 3 pacientes transfundidos en hospitales de otros departamentos $(1,8 \%)$.

Tabla 2. Indicaciones de transfusión de componentes sanguíneos en la población de estudio.

\begin{tabular}{lcc}
\hline \multicolumn{1}{c}{ Motivo de transfusión } & $\begin{array}{c}\text { Mujeres } \\
\mathrm{n}=\mathbf{1 1 6}\end{array}$ & $\begin{array}{c}\text { Hombres } \\
\mathrm{n}=\mathbf{5 0}\end{array}$ \\
\hline Cirugía* $^{*}$ & $51,7 \%$ & $28 \%$ \\
Accidente de tránsito & $2,58 \%$ & $22 \%$ \\
Hemorragia posparto & $12,6 \%$ & $\mathrm{NA}$ \\
Herida por arma de fuego & $2,58 \%$ & $10 \%$ \\
Aborto & $5,17 \%$ & $\mathrm{NA}$ \\
Herida por arma corto punzante & $0,86 \%$ & $4 \%$ \\
Exanguinotransfusión & $5,17 \%$ & $8 \%$ \\
Enfermedades hematológicas & $5,17 \%$ & $2.58 \%$ \\
Enfermedades coagulación & $0,86 \%$ & $2 \%$ \\
Hemodiálisis & $\mathrm{NA}$ & $4 \%$ \\
Otros ${ }^{* *}$ & $7,75 \%$ & $22 \%$ \\
\hline
\end{tabular}

* Cirugía en el caso de mujeres incluye cesárea. NA: No aplica.

** Otros incluye hemorragias del tracto digestivo y quemaduras, entre otros.

Según los resultados de las pruebas serológicas, 11 $(6,6 \%)$ muestras presentaron positividad en la prueba de ELISA para anti-VHC. La detección del genoma viral se logró en 7 de las 11 (63\%) muestras positivas para la prueba de ELISA y corresponde a una frecuencia de detección del genoma del VHC de $63,6 \%$ de las muestras con marcador anti-VHC (tabla 3). Los individuos que presentaron marcadores de infección por el VHC, en su mayoría no tenían ningún factor de riesgo adicional a la transfusión. El análisis no mostró significancia estadística para ninguna de las variables analizadas en los individuos con infección por el VHC (Anexo 1).

Con el fin de evaluar la función hepática de los individuos VHC positivos, se determinó el nivel de transaminasas en suero en 5 de los 7 individuos que resultaron positivos para el genoma de VHC. En 4 pacientes se encontraron valores superiores a los de referencia, como se espera en caso de infección crónica por VHC. No se dispone de otros análisis bioquímicos ni histopatológicos para determinar el grado del compromiso hepático.

El análisis del patrón de corte con enzimas de restricción (RFLP) permitió la identificación del genotipo 1 en 4 de las muestras $(57,1 \%)$. Para comparar el patrón de 
restricción, se realizó un análisis in silico en el programa NebCutter V2.0 utilizando una secuencia colombiana perteneciente al genotipo 1 previamente publicada (GenBank $\mathrm{N}^{\circ}$ : JF693489.1). Para la enzima HaeIII se identificó un sitio de corte en la posición 216 y para la enzima RsaI se identificaron dos sitios de corte en las posiciones $102 \mathrm{y}$ 225. En la figura 1 se muestra el patrón de corte para las enzimas HaeIII y RsaI y un gel representativo del patrón las muestras analizadas. Al comparar el patrón obtenido en 4 de las muestras con los análisis in silico y lo previamente reportado se determinó que correspondían al genotipo 1. En las 3 muestras restantes no fue posible hacer el análisis por muestra insuficiente.

Tabla 3. Factores de riesgo en individuos transfundidos antes de 1994 con marcadores de infección por VHC.

\begin{tabular}{|c|c|c|c|c|c|}
\hline Código & Edad & Género & $\begin{array}{c}\text { Motivo } \\
\text { transfusión }\end{array}$ & Anti-VHC & $\begin{array}{c}\text { Genoma } \\
\text { VHC }\end{array}$ \\
\hline 9 & 56 & $\mathrm{~F}$ & Cirugía & Pos & Pos \\
\hline 15 & 53 & M & Cirugía & Pos & Pos \\
\hline 25 & 38 & $\mathrm{~F}$ & Cirugía & Pos & Neg \\
\hline 30 & 26 & M & $\begin{array}{l}\text { Enfermedades } \\
\text { hematológicas }\end{array}$ & Pos & Pos \\
\hline 33 & 57 & $\mathrm{~F}$ & Cirugía & Pos & Pos \\
\hline 50 & 38 & $\mathrm{~F}$ & Aborto & Pos & Pos \\
\hline 60 & 29 & $\mathrm{~F}$ & Otros* & Pos & Neg \\
\hline 66 & 30 & M & Aborto & Pos & Neg \\
\hline 69 & 41 & $\mathrm{~F}$ & Otros* & Pos & Neg \\
\hline 92 & 29 & $\mathrm{~F}$ & $\begin{array}{l}\text { Enfermedades } \\
\text { de coagulación }\end{array}$ & Pos & Pos \\
\hline 126 & 40 & $M$ & $\begin{array}{l}\text { Accidente por } \\
\text { arma fuego }\end{array}$ & Pos & Pos \\
\hline
\end{tabular}

Resumen de las características de los individuos del estudio que presentaron marcadores de infección por VHC.

F: Femenino, M: Masculino. Pos: Positivo Neg: Negativo.

* Otros incluye hemorragias del tracto digestivo y quemaduras, entre otros.

Anexo 1. Análisis de variables en los individuos del estudio con infección por VHC.

\begin{tabular}{|c|c|}
\hline Variable & Valor $p$ (dos colas) \\
\hline Edad & 0,117 \\
\hline Escolaridad & 0,296 \\
\hline \# Unidades transfundidas & 0,924 \\
\hline Género & 0,642 \\
\hline Hemodiálisis & 0,088 \\
\hline Hospitalización & 0,458 \\
\hline Piercing & 1,0 \\
\hline Tatuajes & 1,0 \\
\hline
\end{tabular}

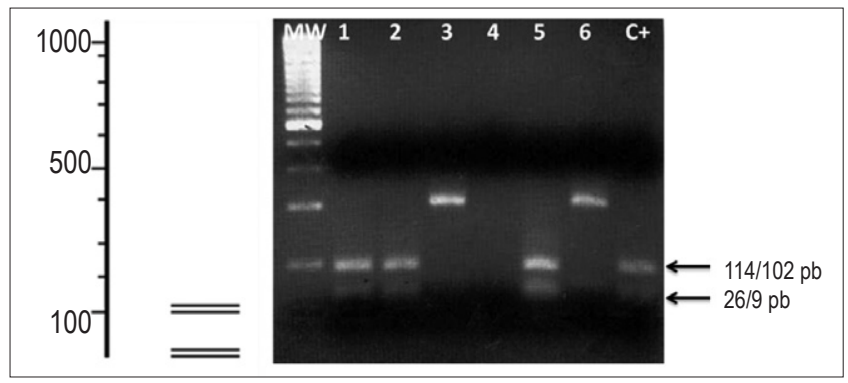

Figura 1. Análisis de genotipos mediante RFLP. Resultado representativo del análisis mediante RFLP de las muestras positivas por RT-PCR. En la figura se observa el patrón de corte de las enzimas de restricción HaeIII/RsaI en la secuencia JF693489.1 Genotipo 1 y un gel representativo del análisis de RFLP en las muestras del estudio. Los carriles 1,2 y 5 corresponden muestras con el patrón de corte del genotipo 1. C+: Control positivo, clon HCV-H77. MW: Marcador de peso molecular de $100 \mathrm{pb}$.

\section{DISCUSIÓN}

El VHC se encuentra ampliamente distribuido a nivel global y se estima que alrededor de $3 \%$ de la población mundial se encuentra infectada (16). En Latinoamérica la prevalencia promedio es de $1,23 \%$, aunque varía entre los países (17).

En Colombia se desconoce la prevalencia de infección en población general, aunque se estiman alrededor de 400.000-500.000 personas infectadas en el país (18). Se han realizados algunos estudios en grupos con factores de riesgo y en donantes de sangre; según un estudio reciente la prevalencia de anti-VHC fue de $0,6 \%$ en 54.499 donantes de sangre en un banco de sangre en Medellín en el periodo 2007-2010 (19).

En el presente estudio se determinó la frecuencia de infección por VHC en una población de individuos que recibieron transfusión de componentes sanguíneos antes de 1994; se analizaron el motivo de transfusión y los factores de riesgo asociados a la infección por VHC adicionales a la transfusión. En un total de 11/166 (6,6\%) de las muestras analizadas se detectaron anticuerpos anti-VHC y fue posible confirmar la presencia del genoma viral en $7 / 11$ muestras mediante RT-PCR. Estos resultados son similares a reportes en pacientes multitransfundidos publicados en países de la región como Honduras, Uruguay, Brasil y Argentina en los cuales se describen prevalencias de antiVHC entre 7 y 16,7\% (19-22).

En el estudio realizado por Beltrán y cols en una población de 500 pacientes multitransfundidos atendidos en cuatro instituciones de Medellín y Bogotá se demostró una prevalencia de infección por VHC de 9\%, según el marcador anti-VHC (23). En esta población de estudio se demostró que el riesgo de infección por VHC disminuyó 
3 veces entre 1993 y 1995, y luego el riesgo disminuyó más de $90 \%$ acorde con la cobertura de tamizaje de $99 \%$ de las unidades de sangre en Colombia después de 1995. Este estudio demostró el impacto de las políticas de salud pública en la medicina transfusional como el tamizaje de $100 \%$ de las unidades, el mejoramiento en la selección de los donantes y el aumento en el número de donantes voluntarios, entre otras medidas (23).

La frecuencia de infección por VHC también ha sido explorada en pacientes colombianos sometidos a hemodiálisis, en usuarios de drogas psicoactivas, en pacientes infectados con el virus de la inmunodeficiencia humana (VIH) y pacientes con enfermedad hepática crónica. El primero corresponde a un estudio prospectivo realizado en 4 unidades de diálisis de la ciudad de Cali en $2007-$ 2008, en el cual se incluyeron 999 pacientes sometidos a hemodiálisis y se determinó una prevalencia de $2,9 \%$ en esta población; de manera importante en este estudio $41,3 \%$ de pacientes con anti-VHC positivos presentaron antecedente de transfusión (24).

El segundo estudio fue realizado en centros de rehabilitación y en un centro de reclusión de la ciudad de Bucaramanga en 2009. En este estudio se reporta una prevalencia de anti-VHC de $0 \%$ en 259 muestras provenientes de individuos que declararon consumo de drogas psicoactivas; la mayoría declaró consumo de drogas vía oral o nasal (98\%) y solo un bajo porcentaje reportó consumo de drogas por vía intravenosa $(4,2 \%)(25)$.

Resultados similares fueron descritos en pacientes con infección por VIH con una frecuencia de anti-VHC de $0,8 \%$; la baja frecuencia descrita en esta población fue atribuida a la ausencia de factores de riesgo como transfusiones y uso de drogas vía endovenosa (26).

También se ha descrito la infección por VHC en pacientes con enfermedad hepática crónica en un estudio prospectivo realizado en un hospital de cuarto nivel de la ciudad de Medellín durante el periodo 2005-2007; en 131 pacientes con diagnóstico de cirrosis hepática y/o carcinoma hepatocelular se demostró una frecuencia de anticuerpos anti-VHC de 6,9\% (27).

De tal manera, el rango de seroprevalencia descrito en los diferentes estudios realizados después de 2005 en poblaciones con factores de riesgo en Colombia es de 0 a $9 \%$. Estos resultados contrastan con lo publicado por AlvaradoMora y cols en una muestra de 176 habitantes de la zona rural y urbana de comunidades indígenas del departamento del Amazonas, con un rango de edad de 12 a 72 años. La frecuencia de anti-VHC en la población de estudio fue de 5,68\% (10/176) (28). Este hallazgo llama la atención según lo descrito recientemente por De la Hoz y cols en 176 muestras de mujeres de comunidades indígenas de la misma zona geográfica del estudio de Alvarado Mora y cols.
La frecuencia de anti-VHC en esta población fue de $0 \%$; es de anotar que las 176 muestras presentaban el marcador de infección por virus de la hepatitis $\mathrm{B}$, anti-HBc (comunicación personal). Como se mencionó anteriormente, el principal factor de riesgo de la infección por VHC en Colombia corresponde a la transfusión sanguínea y las comunidades indígenas son poco expuestas a este riesgo por motivos culturales, lo que no coincide con los resultados de AlvaradoMora y cols. También contrasta con los diferentes estudios realizados en población colombiana con factores de riesgo por VHC (tabla 4). En el estudio de Alvarado y cols también evalúan muestras provenientes de personal de salud, trabajadoras sexuales y/o población desplazada en San Andrés, Chocó y Magdalena; los datos de seropositividad reportados para cada región son $0,66 \%, 3,68 \%$ y $3,87 \%$, respectivamente. Sin embargo, en la publicación los datos no están discriminados por grupo de riesgo (28).

Tabla 4. Seroprevalencia de infección por VHC en diferentes poblaciones en Colombia.

\begin{tabular}{lcl}
\hline $\begin{array}{c}\text { Población de estudiol } \\
\text { Factor de riesgo }\end{array}$ & $\begin{array}{c}\text { Frecuencia } \\
\text { anti-VHC }\end{array}$ & \multicolumn{1}{c}{ Referencia } \\
\hline Trasplante de órganos & $21,5 \%$ & Echavarría, 1992 \\
& $49 \%$ & de la Hoz, 2000 \\
Hemodiálisis & $42,2 \%$ & Echavarría, 1992 \\
& $35-60 \%$ & de la Hoz, 2000 \\
& $6,1 \%$ & Beltrán M et al, 2005 \\
Diagnóstico de hemofilia & $2,9 \%$ & Ramírez et al, 2010 \\
& $6,52 \%$ & Echavarría, 1992 \\
Sangrado agudo & $32 \%$ & de la Hoz, 2000 \\
Diagnóstico de cáncer & $32,2 \%$ & Beltrán M et al, 2005 \\
Infección por VIH & $2,6 \%$ & Beltrán M et al, 2005 \\
& $3,4 \%$ & Beltrán M et al, 2005 \\
Hepatopatías crónicas & $0 \%$ & Hoyos-Orrego et al, \\
& $31 \%$ & 2006 \\
Usuarios drogas psicoactivas & $6,9 \%$ & Cortes et al, 2011 \\
Población indígena, & $0 \%$ & Bautista et al, 2011 \\
anti-HBc+ & $0 \%$ & de la Hoz (comunicación \\
Población indígena & & personal) \\
Donantes de sangre & $5,68 \%$ & Alvarado-Mora et al, \\
& $0,97 \%$ & Echavarría, 1992 \\
& $1,0 \%$ & Beltrán M et al, 1997 \\
& $0,98 \%$ & Cortés A et al, 1999 \\
& $0,47 \%$ & Cortés A et al, 1999 \\
& $0,6 \%$ & Bedoya JAP et al, 2012 \\
Informe Nal de \\
Indicadores 2012
\end{tabular}

Los estudios publicados en Colombia hasta la fecha, junto con los resultados del presente estudio sugieren que la prevalencia del VHC en Colombia es variable depen- 
diendo del grupo de riesgo analizado. Si se tienen en cuenta los resultados desde 2005, en grupos con factores de riesgo como transfusión, hemodiálisis y enfermedad hepática crónica, la prevalencia varía entre $4,2 \%$ y $9 \%$ como lo publicado en otros países de la región $(9,29)$.

Con respecto a la caracterización del genotipo viral, el genoma del VHC se logró amplificar en 7 (63\%) del total de 11 muestras que presentaron anticuerpos anti-VHC. Adicionalmente, se identificó el genotipo 1 en 4 de las muestras mediante análisis de RFLP. Hasta la fecha, los estudios de caracterización molecular publicados han demostrado que el genotipo 1 es el más prevalente en el país. En pacientes con cirrosis hepática y carcinoma hepatocelular se ha descrito exclusivamente la presencia del genotipo 1 del VHC (27).

En un estudio de 35 secuencias del VHC obtenidas de donantes de sangre de la ciudad de Bogotá, se identificó el subgenotipo $1 \mathrm{~b}$ de VHC en $82,8 \%$ de las muestras y en $5,7 \%$ subgenotipo $1 \mathrm{a}$; los subgenotipos $2 \mathrm{a}, 2 \mathrm{~b}$ y $3 \mathrm{a}$ también fueron caracterizados en las muestras de donantes (30). En el estudio de pacientes multitransfundidos citado anteriormente, se caracterizaron 12 secuencias de VHC y se confirmó la presencia del subgenotipo $1 \mathrm{~b}$ en 8 muestras $(66,7 \%)$ y subgenotipo la en 2 muestras (16,7\%); también fueron identificados los subgenotipos $2 \mathrm{~b}$ y $3 \mathrm{a}$ en una muestra en cada caso (31). De los 8 aislados de VHC subgenotipo 1 b, 7 correspondían a pacientes que habían recibido su primera transfusión antes de 1986. Este dato coincide con la estimación realizada por Alvarado-Mora y cols, quienes postularon que la introducción del VHC (subgenotipo $1 \mathrm{~b}$ ) en Colombia (Bogotá) fue aproximadamente en 1950, con un crecimiento exponencial entre 1970 y 1990 , probablemente a través de transfusiones sanguíneas (30).

Los resultados del presente estudio aportan al conocimiento de la epidemiología de la infección por VHC en Colombia en individuos transfundidos antes de 1994. La frecuencia de infección por VHC y el genotipo 1 descrito en esta población es similar a lo reportado en otros países de la región. Sin embargo, se deben realizar estudios adicionales en individuos transfundidos antes de 1996 para el manejo de los casos y la identificación de los candidatos a tratamiento.

\section{Agradecimientos}

Los autores agradecen a Merck Sharp \& Dohme Colombia y a la vicerrectoría de investigación de la Universidad de Antioquia (Proyecto Sostenibilidad) por la financiación para la realización de este trabajo.

\section{REFERENCIAS}

1. Who IN, States M. Global policy report on the prevention and control of viral hepatitis in WHO Member States 2013.

2. Hajarizadeh B, Grebely J, Dore G. Epidemiology and natural history of HCV infection. Nat Rev Gastroenterol Hepatol 2013; 10: 553-62.

3. Perz JF, Armstrong GL, Farrington L, Hutin Y, Bell BP. The contributions of hepatitis $B$ virus and hepatitis $C$ virus infections to cirrhosis and primary liver cancer worldwide. J Hepatol 2006; 45: 529-38.

4. De Martel C, et al. Global burden of cancers attributable to infections in 2008: a review and synthetic analysis. Lancet Oncol 2012; 13: 607-15.

5. Tornesello ML, et al. Mutations in TP53, CTNNB1 and PIK3CA genes in hepatocellular carcinoma associated with hepatitis B and hepatitis C virus infections. Genomics 2013; 102: 74-83.

6. Aghemo A, Colombo M. Hepatocellular carcinoma in chronic hepatitis C: from bench to bedside. Semin. Immunopathol 2013; 35: 111-20.

7. Castello G, Scala S, Palmieri G, Curley S, Izzo F. HCV-related hepatocellular carcinoma: From chronic inflammation to cancer. Clin Immunol 2010; 134: 237-50.

8. Fassio E, et al. Etiology of hepatocellular carcinoma in Latin America: a prospective, multicenter, international study. Ann Hepatol 2010; 9: 63-9.

9. Kershenobich D, et al. Trends and projections of hepatitis C virus epidemiology in Latin America. Liver Int 2011; 31(Suppl. 2): 18-29.

10. Beltrán M, Raad J, Ayala M, Ching R. Tamizaje de enfermedades infecciosas en bancos de sangre, Colombia, 1995. Biomédica 1997; 17: 137-142.

11. Cortés $A B$, Beltrán $M$, Olaya $B$, Hernández $M$. Riesgo de enfermedades infecciosas transmitidas por transfusión en el Valle del Cauca, Colombia. Colomb Med 1999; 30: 13-18.

12. Informe nacional de Indicadores. Red Nacional de Bancos de Sangre y Servicios Transfusionales. Instituto Nacional de Salud. 2012.

13. Chomczynski P, Sacchi N. Single-Step Method of RNA Isolation by Acid Guanidinium Extraction. Anal. Biochem 1987; 162: 156-159.

14. Chan SW, et al. Analysis of a new hepatitis $C$ virus type and its phylogenetic relationship to existing variants. J. Gen. Virol 1992; 73: 1131-41.

15. Davidson F, et al. Survey of major genotypes and subtypes of hepatitis $\mathrm{C}$ virus using RFLP of sequences amplified from the 5' non-coding region. J Gen Virol 1995; 76: 1197-204.

16. Mohd Hanafiah K, Groeger J, Flaxman AD, Wiersma ST. Global Epidemiology of hepatitis C Virus infection: New estimates of age-specific antibody to hepatitis $\mathrm{C}$ virus seroprevalence. Hepatology 2013; 57: 1333-1342. 
17. Méndez-Sánchez N, Gutiérrez-Grobe Y, Kobashi-Margáin RA. Epidemiology of HCV infection in Latin America. Ann Hepatol 2010; 9: 27-29.

18. De la Hoz F. Epidemiología de la hepatitis $C$ en Latinoamérica y Colombia. Biomédica 2000; 20: 66-72.

19. Vinelli E, Lorenzana I. Transfusion-transmitted infections inmulti-transfused patients in Honduras. J Clin Virol 2005; 34 (Suppl. 2): S53-60.

20. López L, et al. Risk factors for hepatitis B and C in multitransfused patients in Uruguay. J Clin Virol 2005; 34 (Suppl. 2): S69-74.

21. Paula EV, et al. Transfusion-transmitted infections among multi-transfused patients in Brazil. J Clin Virol 2005; 34 (Suppl. 2): 27-32.

22. Remesar M, Gamba C, Kuperman S, Marcos M A. Antibodies to hepatitis $\mathrm{C}$ and other viral markers in multitransfused patients from Argentina. J Clin Virol 2005; 34 (Suppl. 2): 20-26.

23. Beltrán, M. et al. Hepatitis $C$ virus seroprevalence in multitransfused patients in Colombia. J. Clin. Virol 2005; 34 (Suppl. 2): 33-38.

24. Ramírez R, et al. Prevalencia de anticuerpos contra el virus de hepatitis $\mathrm{C}$ en unidades de diálisis de Cali-Colombia Prevalence of anti-HCV antibodies among patients on dialysis in Cali-Colombia. Rev Col Gastroengerología 2010; 14-18.
25. Amorocho HB, Zorelly B, Moreno J. Ausencia de infección por virus de la hepatitis $\mathrm{C}$ en usuarios de drogas ilícitas en la ciudad de. Rev Col Gastroengerología 2011; 15-20.

26. Hoyos-Orrego A, Massaro-Ceballos M, Ospina-Ospina M, Gómez-Builes C, Vanegas-arroyave N. Serological markers and risk factors for hepatitis $b$ and $c$ viruses in patients infected with human immunodeficiency virus. Rev Inst Med trop S Paulo 2006; 48: 321-326.

27. Cortes-Mancera F, et al. Etiology and Viral Genotype in Patients with End-Stage Liver Diseases admitted to a Hepatology Unit in Colombia. Hepat Res Treat 2011; 363205.

28. Alvarado-Mora MV, et al. Hepatitis B (HBV), hepatitis C (HCV) and hepatitis delta (HDV) viruses in the Colombian population--how is the epidemiological situation? www.plosone.org. 2011.

29. Szabo SM, et al. The epidemiologic burden of hepatitis C virus infection in Latin America. Ann Hepatol 2012; 11 : 623-35.

30. Alvarado-Mora MV, Gomes-Gouve MS. Molecular Characterization, Distribution, and Dynamics of Hepatitis C Virus Genotypes in Blood Donors in Colombia. J Med Virol 2010; 1898: 1889-1898.

31. Di Filippo D, et al. Molecular characterization of hepatitis c virus in multi-transfused Colombian patients. Virol J 2012; 9: 242 . 


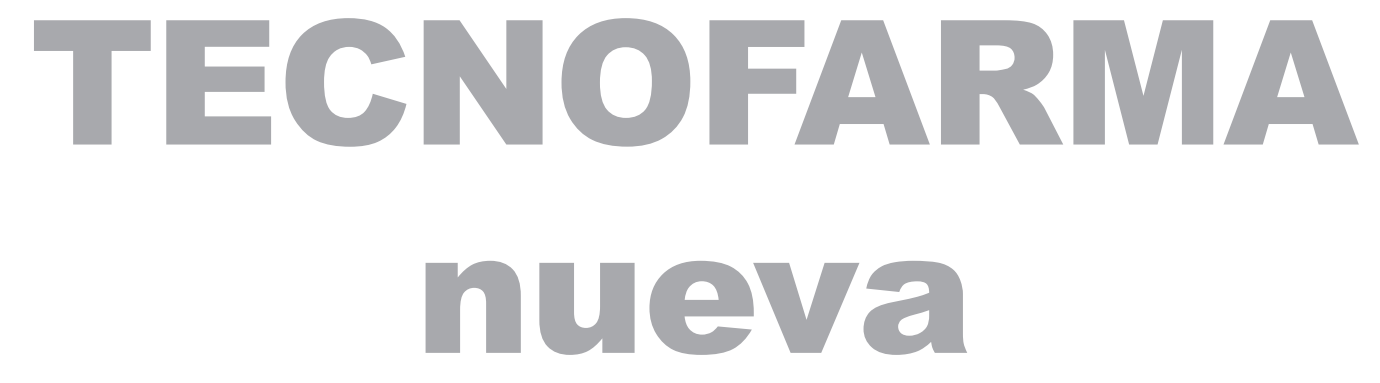

